\title{
Evaluaciones Psicológico-Forenses en asuntos de Violencia de Género. Errores habituales y propuestas de mejora
}

\section{Forensic Psychological Evaluation in cases of Gender- Based Violence. Common errors and proposals for improvement}

Fecha de recepción: 31/05/2016

Fecha de aceptación: 15/07/2016
Laura Fátima Asensi Pérez

Universidad de Alicante

PDI en el Deptartamento de Psicología de la Salud de la Universidad

de Alicante. Miembro del L.O.P.F.(Listado Oficial de Psicólogos

Forenses) del Colegio Oficial de Psicólogos de la Comunidad Valenciana.

Miguel Díez Jorro

Universidad de Alicante

PDI en el Deptartamento de Psicología de la Salud de la Universidad de Alicante. Miembro del L.O.P.F.(Listado Oficial de Psicólogos Forenses) del Colegio Oficial de Psicólogos de la Comunidad Valenciana.

\section{resumen/alostract:}

Este trabajo consiste en una "revisión de campo" y una recopilación de errores habituales detectados en evaluaciones psicológico-forenses en casos de violencia de género. A lo largo de nuestra experiencia profesional como psicólogos forenses y en nuestra práctica habitual encontramos dictámenes e informes en este ámbito que adolecen, en muchos casos, de rigor metodológico, dejando patente escaso conocimiento sobre la violencia de género, sus manifestaciones, evolución, victimización y consecuencias, llegando a interpretaciones y conclusiones periciales, cuanto menos, inexactas e incluso en ocasiones iatrogénicas. Los errores evaluativos que se han detectado condicionarán recomendaciones y discusiones periciales que se no ajustan a la realidad de los asuntos valorados, lo que pudiera provocar no solo efectos de victimización secundaria de mujeres víctimas y niños/as, sino que, además, los informes periciales psicológicos no cumplirán con su función básica de asesoramiento especializado y auxilio judicial. La propia exposición realizada en este trabajo de errores evaluativos y de interpretación conlleva inherentemente diferentes propuestas de mejora, entre las que destacamos formación especializada, el uso de protocolos de evaluación adecuados así como la realización de informes periciales estructurados y adaptados al contexto judicial.

This work consists in a "field review" and a common mistakes compilation detected in psychological-forensic assessments in events of gender-based violence. In our professional experience being forensic psychologist and our daily job, we have found opinions and reports that, in many cases, suffer from methodological rigor, restricting the knowledge ofintimate partner violence, their manifestations, evolution, victimization and consequences, leading to inaccurate and iatrogenic interpretations and conclusions. The evaluative mistakes detected will determine recommendations and expert discussions that could not be real about these complicated affairs, that could cause not only secondary victimization effects in women and children but also the psychological expert reports will not fulfil their basic function of expert advice and legal aid. This explanation about interpretation and evaluative mistakes leads to different proposals of improvement, specialized training, the use of appropriate assessment protocols and to do expert, structured and adapted reports to judicial context. 


\section{palabras clave/keywords:}

Evaluación psicológica, Violencia de género, Informe pericial, Psicología forense, Maltrato habitual, Maltrato psicológico, Abuso emocional

Psychological evaluation, Intimate partner violence, Expert testimony, Forensic psychology,

Habitual abuse, Psychological abuse

\section{INTRODUCCIÓN}

Éste es un trabajo básicamente de campo y consiste en una recopilación por parte de los autores de errores habituales encontrados en evaluaciones psicológico-forenses en procedimientos de violencia de género.

En la práctica de los tribunales el dictamen de peritos es uno de los medios de prueba de mayor relevancia, como viene a demostrar el estudio de la jurisprudencia y su frecuente utilización cuando es necesario realizar análisis o estudios técnicos sobre el objeto del proceso y los hechos que lo conforman (Sánchez, 2014; Cárdenas, 2011). Atendiendo a la función principal de asesoramiento judicial, entendemos que evaluaciones sin evidencia científica, siguiendo una metodología de escaso valor científico y con interpretaciones e inferencias subjetivas, no suministrarán al juzgador argumentos válidos para la formación de su convencimiento.

Realizar evaluaciones psicológicas forenses con ausencia de rigor y de fundamento teórico, utilizando metodología inapropiada, con desconocimiento del fenómeno de la violencia de género y de las manifestaciones del maltrato psicológico, causas, evolución, mantenimiento, victimización, indicadores de riesgo, etc., puede llevar a interpretaciones, análisis y conclusiones forenses inválidas e incluso iatrogénicas. Los errores evaluativos condicionarán recomendaciones y discusiones periciales que se no ajustan a la realidad de los asuntos valorados, lo que pudiera provocar no solo efectos de victimización secundaria, sino que además los informes periciales no cumplirán con su función básica de asesoramiento especializado y auxilio judicial.

Además de incidir en la necesidad de utilidad y eficacia de las evaluaciones psicológicas en el contexto forense-judicial, también consideramos necesario evitar en lo posible la revictimización de las mujeres y sus hijos/as víctimas de la violencia de género. El daño psíquico padecido por las víctimas de malos tratos, más la vulnerabilidad especial de estas víctimas, precisamente por su exposición a maltrato continuado, lleva a que sea fácil reforzar su victimización en una relación asistencial, jurídica o profesional (policial, médica, jurídica, psicológica, social, etc.). Al hilo de lo anterior, en la nueva Ley del Estatuto de la Víctima (Ley 4/2015) que se encuentra en vigor desde 28 de octubre de 2015, se establecen normas mínimas sobre los derechos, apoyo y protección de las víctimas de delitos. Consiste en un nuevo régimen jurídico para avanzar y mejorar la respuesta a sus necesidades e intereses legítimos en el marco del proceso penal, con una especial atención a los colectivos más vulnerables para prevenir su victimización secundaria. El Estatuto diferencia entre las víctimas directas e indirectas (familiares, hijos...) y tiene en cuenta el aspecto moral de las mismas (Vidal, 2015).

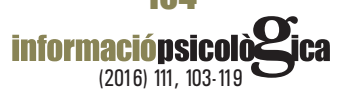


Como peritos y forenses se deberá estimar a lo largo de nuestro ejercicio profesional, sin tener por qué considerarse dejación de funciones, la protección de las víctimas de violencia de género (considerando también a los menores expuestos) de forma más integral y ajustada a los principios y a las metas establecidas en las Directivas Europeas y el Convenio de Estambul.

Describiremos a lo largo de este trabajo aspectos relacionados con la violencia de género en particular, detallaremos diferentes mecanismos de abuso emocional o maltrato psicológico que por su "invisibilidad" pueden pasar inadvertidos a evaluadores con escasa formación y/o experiencia. Destacamos, centrándonos a nivel victimológico, las mujeres como víctimas directas y también a los menores expuestos a violencia de género, como víctimas de riesgo directas e indirectas, especialmente vulnerables. Posteriormente comentamos algunos aspectos que consideramos importantes acerca de los peritajes psicológicos, para, finalmente, centrarnos en los errores más comunes que hemos detectado en nuestra práctica profesional forense en evaluaciones sobre violencia de género y asuntos afines.

\section{DESCRIPCIÓN Y PROCEDIMIENTO}

El procedimiento de este trabajo ha consistido en el estudio de expedientes judiciales que incluían informes psicológicos evaluativos en asuntos relacionados con la violencia de género. Se han recogido tanto informes escritos de evaluación psicológica como informes psicológico-forenses que constaban en autos. Nos hemos centrado en aquellos errores más habituales que hemos observado en diferentes informes periciales a los que hemos tenido acceso en el estudio de expedientes judiciales para el desarrollo de nuestra práctica forense.

Este texto es fruto y ha nacido, por tanto, de la experiencia propia de sus autores. El objetivo de este trabajo no es otro que ofrecer una visión sobre la realidad práctica actual de los peritajes psicológicos en el contexto de la violencia contra la mujer y en otros asuntos judiciales relacionados. Esta disquisición es una ampliación de un somero trabajo de observación que realizamos hace ocho años (Asensi y Díez, 2008) y el análisis longitudinal nos conduce a pensar que es necesario insistir en propuestas de mejora en cuanto a la calidad y eficacia de la emisión de informes psicológicos en estos asuntos.

\section{CONCEPTOS BÁSICOS REFERIDOS A LA VIOLENCIA}

El Informe sobre la situación mundial de la prevención de la violencia de la OMS (2014) refiere que "a pesar del elevado número de muertes resultantes de la violencia y la vasta escala en que las consecuencias no mortales de la violencia afectan a las mujeres, los niños y las personas mayores, los datos presentan lagunas importantes que socavan las iniciativas para prevenirla"

Andrés Pueyo (2012) afirma que la violencia "es un fenómeno que tiene los mismos efectos que la enfermedad: dolor, lesiones, sufrimiento y muerte. Pero la violencia, a diferencia de la enfermedad, resulta de una acción intencionada del hombre que provoca el malestar y el sufrimiento de las víctimas". La violencia, en cualquier escenario, tiene un doble efecto sobre la víctima, produciéndole efectos perniciosos tanto a nivel físico como a nivel psíquico, así como consecuencias adaptativas y sociales.

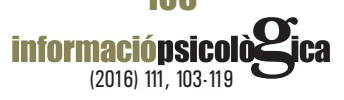




\section{La Violencia de género}

La violencia de género es conceptualizada por la Ley Orgánica 1/2004 de Medidas de Protección Integral contra la Violencia de Género: "una violencia que se dirige sobre las mujeres por el hecho mismo de serlo, por ser consideradas por sus agresores carentes de los derechos mínimos de libertad, respeto y capacidad de decisión y que tiene como resultado un daño físico, sexual o psicológico". Se incluye las agresiones a la libertad sexual, las amenazas, las coacciones o la privación arbitraria de libertad. Recientemente este tipo de violencia está dejando de ser considerada un asunto privado y cobra relevancia de problema social que debe ser comprendido, prevenido y adecuadamente valorado.

Se consideran tres tipos de violencia que se interrelacionan dentro de las situaciones de malos tratos (Labrador, 2004): el maltrato físico, el maltrato psicológico y el maltrato sexual, cuya severidad y frecuencia varían de una situación a otra, pero cuyo objetivo común es el control de la víctima. La Organización de las Naciones Unidas (2014) recomienda considerar los siguientes tipos básicos de violencia contra la mujer: la violencia física, la violencia sexual, la violencia psicológica y la violencia económica.

Novo y Seijo (2009) afirman que la gravedad de las consecuencias de la victimización en estos asuntos se relaciona con la intensidad del maltrato, encontrando que la violencia psicológica también actúa como un buen predictor del Trastorno por Estrés Postraumático. Las mujeres víctimas de violencia por parte de sus parejas o ex parejas desarrollan un alto nivel de deterioro global y problemas graves de adaptación a las diferentes áreas vitales. Labrador, Fernández-Velasco y Rincón (2010) señalan en su estudio sobre mujeres víctimas de violencia de pareja que un $44 \%$ de estas mujeres presentan antecedentes de ideación suicida o intento autolítico. Coincidimos en resaltar la necesidad de evaluar y/para prevenir dicho riesgo.

Destacamos algunos datos relacionados con la violencia contra la mujer (OMS, 2016) a fin de determinar la importancia, magnitud y complejidad de este fenómeno. Se considera que la violencia -especialmente la ejercida por su pareja y la violencia sexual- constituye un grave problema de salud pública y una violación de los derechos humanos de las mujeres. Por otra parte, las cifras recientes de la prevalencia mundial indican que alrededor de una de cada tres mujeres en el mundo han sufrido violencia física y/o sexual de pareja. Un $38 \%$ de los asesinatos de mujeres que se producen en el mundo son cometidos por su pareja. Cabe señalar que la realidad sobre la incidencia de este tipo de violencia se ve obstaculizada por la gran ocultación social. Es por ello que no todos los casos en los que existe violencia son reflejados a través de los indicadores judiciales y epidemiológicos (Novo y Seijo, 2009).

En la Macroencuesta de Violencia contra la Mujer 2015 de la Delegación Gobierno para la Violencia de Género del Ministerio del Interior de España se destacan las consecuencias físicas y psíquicas de la violencia de género con un alto nivel de percepción de los efectos de la violencia sufrida por las víctimas sobre su bienestar físico o mental, en cualquiera de sus manifestaciones. El informe relativo a dicha encuesta refleja que los sentimientos de las mujeres que han sufrido violencia física, sexual o miedo de alguna pareja o expareja tras los episodios de violencia, son, en orden de prevalencia: 
- Impotencia ante la situación: 60,7\%.

- Tristeza: $59,8 \%$.

- Rabia: $58,4 \%$.

- Miedo: 51,6\%.

- Angustia: 49,9\%.

- Vergüenza: $38,7 \%$.

- Culpa: 30,1\%.

- Agresividad: 19,1\%.

\section{Violencia psicológica o violencia invisible}

Describir la violencia psicológica como manifestación de malos tratos en situaciones de violencia de género es básico para comprender la importancia de considerar este aspecto en las evaluaciones psicológico-forenses, teniendo presente tanto el proceso violento sin lesiones psíquicas, como las consecuencias emocionales en las víctimas de malos tratos psicológicos continuados. En el contexto de la violencia de género, las agresiones físicas habitualmente producen consecuencias psicológicas. Asimismo, se puede dar, únicamente, la violencia psicológica, pudiendo provocar numerosas secuelas tanto a nivel físico como emocional. Este tipo de violencia "invisible" puede causar en la víctima trastornos psicológicos, desestructuración psíquica, agravar enfermedades físicas o, incluso, provocar el suicidio (Lorente, Sánchez de Lara y Naredo, 2006; Labrador, Fernández-Velasco y Rincón, 2010).

Entendemos, por tanto, la violencia psicológica, en aras a su evaluación, tanto como proceso violento en sí mismo, como efecto de cualquier tipo de agresión violenta física o psíquica (Asensi, 2008).El maltrato continuado genera en la mujer un proceso patológico de adaptación denominado por Walker (1989) "Síndrome de la mujer maltratada". Más recientemente Walker (2012) incorpora nuevas variables y presenta datos más actuales sobre este síndrome (constituyendo un recurso sumamente valioso para los profesionales) realizando una revisión muy detallada acerca del estrés postraumático y la revivencia del trauma; el nivel de ansiedad y excitación; la conducta elusiva y la depresión; las perturbaciones en las relaciones interpersonales; la distorsión de la imagen corporal y las enfermedades físicas; los problemas sexuales y los sentimientos de culpabilidad, vergüenza y celos. Ante el maltrato se desarrolla lo que la autora describe como "impotencia aprendida" por la cual las mujeres mantienen la relación con el maltratador y no ponen fin al abuso.

La realidad nos muestra que ejercer control sobre la pareja es una cuestión que trasciende a la mera agresión física, cuya expresión en la relación es producto del incremento progresivo de patrones de interacción coactiva aparecidos en etapas precedentes a la consolidación de la pareja, es decir, durante el noviazgo (Pozueco, Moreno, Blazquez y García, 2013).

En cuanto a los indicadores de maltrato psicológico podemos distinguir mecanismos encubiertos y mecanismos manifiestos de abuso emocional que conforman una violencia invisible pero no por ello menos lesiva para la víctima. Porrúa et al. (2010) ofrecenuna nueva e

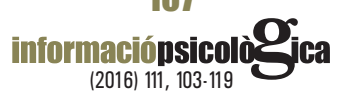


interesante propuesta de clasificación de las diferentes estrategias de abuso psicológico en la pareja partiendo de una perspectiva psicosocial (estableciendo seis categorías y veinte subcategorías) estableciendouna jerarquización de las mismas. Esta categorización jerárquica serviría como guía para orientar a los profesionales que realizan evaluaciones psicológicas forenses si es de aplicación o no el abuso psicológico en el ámbito de la violencia de pareja del caso concreto objeto de valoracióny, en su caso, evaluar posteriormente sus consecuencias.

Consideramos, en definitiva, violencia psicológica como una conducta que causa un perjuicio, aunque pueda estar oculta o disimulada. En este sentido, desde el punto de vista psicológico-forense, se debe valorar y en su caso constatar la existencia de maltrato psicológico en sus diferentes manifestaciones, utilizando para ello diferentes estrategias evaluativas, protocolos validados, modelos multimétodo y considerando la consistencia interevaluador.

\section{Menores víctimas de la violencia de género}

En las evaluaciones forenses en asuntos de violencia de género se debe considerar la posibilidad de que haya víctimas menores que también sufren la exposición o maltrato directo en este contexto. Sería por tanto un error obviar a los niños y niñas que forman parte del núcleo familiar donde ha habido violencia contra la mujer por parte de su pareja o ex pareja. En este trabajo exponemos la necesidad, desde el contexto forense, de evaluar adecuadamente a los menores expuestos a violencia de género, pudiendo considerarse a los mismos como víctimas de riesgo, directas o indirectas, y víctimas especialmente vulnerables.

Numerosos estudios informan de diferentes problemas conductuales y emocionales en niños y niñas víctimas y testigos de violencia de género. Alcántara, López-Soler, Castro, y López (2013) afirman que los datos obtenidos avalan la magnitud y gravedad de las consecuencias de la exposición de los menores a violencia de género; "en la mayoría de los síndromes analizados, la prevalencia clínica de éstos es cinco veces superior a la registrada en población normal, y en algún síndrome hasta diez veces superior". Diferentes variables intervienen en la determinación del impacto emocional y la psicopatología consecuente (Olaya, Tarragona, de la Osa Chaparro y Ezpeleta, 2008) influyendo asimismo en la adaptación de los menores la concurrencia de diversos tipos de violencia (Kinard, 2004), aspectos que el evaluador deberá tener en cuenta.

Por otra parte, y teniendo en consideración la especial vulnerabilidad de los menores, en el artículo 23 de la Ley del Estatuto de la Víctima del Delito se establece la necesidad de realizar una evaluación individual de las víctimas a fin de determinar sus necesidades especiales de protección. Esta valoración considerará especialmente las características personales: si se trata de víctimas menores de edad o en las que concurran factores de especial vulnerabilidad, la naturaleza del delito, la gravedad de los perjuicios o el riesgo de reiteración del delito. En base a lo anterior, en las evaluaciones forenses habrá que tener una especial atención a los colectivos más vulnerables y el proceso de victimización del que han sido objeto.

Estimamos de especial importancia en la evaluación forense de los menores en estos asuntos -como probables víctimas directas e indirectas- aplicar un protocolo de evaluación psicológica forense adaptado ad hoc (Asensi, 2014; Asensi y Díez, 2015).

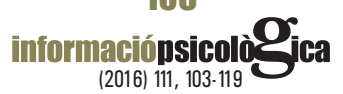




\section{EVALUACIONES PERICIALES PSICOLÓGICAS EN VIOLENCIA DE GÉNERO}

Podemos considerar la evaluación psicológica jurídico-forense como la exploración de todos los aspectos relevantes, positivos o negativos, de un sujeto o sujetos (encausados, víctimas y/o testigos) con el objetivo de responder a las demandas que se realizan desde el ámbito legal, y de esta forma contribuir a la toma de decisiones respecto de la conducta delictiva y sus implicaciones.Se deberá valorar la información relevante al caso que nos ocupa y en función de la solicitud pericial, a fin de auxiliar a los juzgadores en la clarificación del conflicto y en su toma de decisiones.

El informe pericial se emite para constatar, a través de una valoración técnica, una realidad no perceptible. Su fin es hacer visible lo invisible, tangible lo intangible. Como afirma Jouvencel(2003) el forense realizará un informe de valoración psicológica, despojada de valoración jurídica, que deberá integrarse en el conjunto del asunto judicial. Desde esta perspectiva, el psicólogo ofrece al tribunal una versión de los hechos que se convierte en demostrada y veraz (Col.legi Oficial de Psicologia de Catalunya, 2014).

Una de las cuestiones más difíciles con la que se enfrenta el sistema judicial en los casos de violencia de género es la prueba de los hechos que constituyen la misma, ya que en la mayoría de los casos solo se cuenta con la declaración de la víctima. La red de factores que explican el desencadenamiento y reproducción de la violencia es ciertamente compleja. No obstante, su transmisión, manifestaciones y efectos siguen ciertas reglas que se estudian desde diversos ámbitos del conocimiento (Larizgoitia, 2006). La dificultad en el contexto forense estriba en evaluar, interpretar y explicar este tipo de violencia como una violencia también emocional, mitologizada, cuyos efectos necesitan quedar esclarecidos y desvelados ante los operadores del ámbito judicial.

Entendemos que un protocolo de evaluación validado empíricamente resultaría un procedimiento que resolvería problemas, errores y dificultades en la realización de informes y dictámenes en asuntos relacionados con la violencia sobre la mujer. Un protocolo fiable y científicamente avalado debe tener en cuenta las principales áreas de valoración (Asensi, 2008). Navarro Góngora, Navarro Abad, Vaquero y Carrascosa (2004) proponen tres aspectos a valorar: en primer lugar, establecer que el maltrato y la violencia psicológica ha tenido lugar, en segundo lugar, valorar las consecuencias psicológicas (lesión psíquica o secuelas) de dicho maltrato y, por último, establecer y demostrar el nexo causal entre la situación de violencia y el daño psicológico (lesiones psíquicas y secuelas emocionales). Desde nuestra profesión y experiencia consideramos asimismo la necesidad de valorar/descartar indicios de simulación y evaluar, en su caso, indicadores de exactitud y de credibilidad de los testimonios.

Arce y Fariña (2015) han creado un protocolo de ejecución global adaptado a la práctica forense, el Sistema de Evaluación Global, que integra unas fases adaptadas a casos de violencia de género. El SEG consiste en un sistema categorial metódico (Arce, 2010). Los pasos que proponen los autores incluyen la obtención del testimonio y su repetición, el estudio de la motivación, el análisis de la realidad de las declaraciones, fiabilidad de las medidas, medida de la secuela psicológica, evaluación del testimonio de los testigos, análisis de las

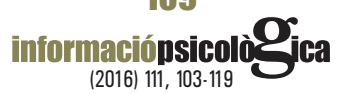


características psicológicas e implicaciones para la redacción del informe pericial. Entendiendo de sumo interés el conocimiento de dicho protocolo, y por no abundar más en el mismo, invitamos a profundizar acudiendo a las publicaciones de los propios autores.

\section{ERRORES MÁS FRECUENTES EN LAS EVALUACIONES PSICOLÓGICAS DE VIOLENCIA DE GÉNERO EN EL CONTEXTO FORENSE}

Nuestra experiencia profesional como peritos forenses nos ha llevado a detectar algunos errores habituales en los peritajes de asuntos referidos a violencia de género que son debidos, básicamente, a problemas metodológicos, diagnósticos y de interpretación de los hechos valorados.En algunos casos también encontramos que existen errores condicionados por la solicitud de los dictámenes periciales por parte de las diferentes instancias judiciales.

\section{1) Errores metodológicos}

1.1 No utilizar un protocolo adecuado.

La evaluación en Psicológica Forense se debe realizar mediante un protocolo adecuado a los hechos evaluados y debe de incluir una estrategia redundante, un modelo evaluativo multimétodo, entrevistas apropiadas de corte clínico-forense, establecer nexos causales, utilizar diversidad de instrumentos a fin de mejorar la fiabilidad y consistencia intermedidas, ytambién considerar la consistencia interevaluador a fin de poder integrar la información relevante y concluir con fundamento.Nos remitimos a los diferentes protocolos referidos en este mismo trabajo como punto de partida para una evaluación forense eficaz.

1.2 La utilización de pruebas psicológicas inadecuadas y/o irrelevantes para la evaluación de maltrato, 0 una utilización incorrecta de tests psicométricos.

El grado de fiabilidad que puede merecer un dictamen pericial vendrá ligado a los elementos y datos que el perito hubiera seleccionado para emitir su opinión técnica, así como su especialidad y comprensión del proceso. Ejemplos a propósito de este tipo de error evaluativo, encontramos: evaluar la capacidad intelectual de la víctima de forma aislada y sin indicios de deterioro cognitivo; antecedentes biográficos irrelevantes; antecedentes médicos de familiares y allegados; uso de pruebas con escasa validez y fiabilidad diagnóstica, abuso de pruebas psicométricas no relevantes al caso o, por el contrario, basarse únicamente en el juicio clínico.

Cuando se utilizan test, sus características y propiedades psicométricas deben ser muy exigentes en el contexto forense, ya que se van a tomar decisiones y realizar recomendaciones importantes sobre las vidas de personas. "Para llevar a cabo una evaluación psicológica rigurosa es necesario que los profesionales que la realizan tengan una preparación adecuada, que los tests utilizados muestren unas buenas propiedades psicométricas, y que se utilicen de forma correcta" (Muñiz, Hernández y Ponsoda, 2015). Entendemos que conocer las nuevas directrices sobre su uso de la Comisión Internacional de Tests constituye un aspecto imprescindible, como afirman los autores "no es suficiente que un test tenga las propiedades psicométricas adecuadas y que los profesionales que lo utilizan tengan una buena cualificación, además hay que garantizar que se hace un uso adecuado de las pruebas, y a ello van encaminadas las directrices descritas".

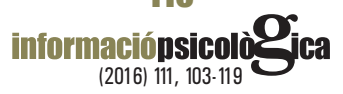




\subsection{Utilización de entrevistas de corte clínico en evaluación forense}

La entrevista de corte clínico, entre otras cuestiones, está enfocada hacia un/a paciente, da pistas sobre la sintomatología y suele estar enfocada a una posterior intervención. La entrevista forense, por el contrario, tiene un objetivo determinado, está enfocada a unos hechos concretos, maneja diferentes hipótesis, tiene una estructura particular, es imparcial y se aleja del enfoque clínico tradicional, de manera que aporta una justa y correcta toma de decisiones en los sistemas judiciales. La entrevista forense está orientada a la reinstauración de contextos, enfocada a valorar la huella psíquica y la sospecha de posible simulación. Existen modelos de entrevista de uso adecuado en el ámbito judicial y protocolos adaptados a la evaluación forense.

\subsection{Realizar las entrevistas en formato de interrogatorio, con preguntas capciosas y/o sugestivas.}

La forma en que se realizan las preguntas puede influir en la memoria del sujeto, en las respuestas dadas, minimiza la objetividad y dificulta el recuerdo libre. Es por ello que no se deben realizar durante la evaluación pericial preguntas con las respuestas implícitas o preguntas sugerentes que pueden perjudicar la huella de la memoria y fomentar la obtención de información inválida.

La entrevista en formato interrogatorio, por otra parte, no es válida ni eficaz para realizar una valoración en el contexto forense (Arce y Fariña, 2012) por la posibilidad de contaminar la prueba y limitar la cantidad y calidad de la información ofrecida por la víctima. Las entrevistas clínico-forenses en formato narrativo son más eficaces, pudiendo aplicarse en fases, por lo que son útiles para la evaluación de maltrato continuado, ajustándose a la posibilidad de realizar un análisis de realidad.

Un protocolo sistematizado para analizar y seleccionar la información del expediente judicial es otro aspecto importante que puede condicionar el tipo de entrevista, las preguntas adecuadas al caso que se está evaluando y la información relevante a estimar. Todo ello con el fin de conocer de forma exhaustiva los antecedentes y el asunto en su conjunto. Consiste en realizar un estudio y análisis del expediente en autos, generar diferentes hipótesis y planificar la entrevista "cumpliendo las máximas del buen entrevistador" (Scott y Manzanero, 2015).

1.5 Recabar más información de la necesaria e irrelevante para el caso, con el consiguiente sufrimiento y posibles perjuicios para la persona evaluada.

El perito forense debe centrarse en aquella información que resulte relevante para realizar su evaluación del caso particular y no en datos que resulten irrelevantes y no aporten información significativa. Todo ello por evitar perjuicios innecesarios, también por economía temporal, evitar errores de atribución, obtención de exceso de información confusa e improcedente ya queun exceso de información irrelevante puede interferir en la interpretación global de los hechos evaluados.

\subsection{Apriorismo confirmatorio}

Consiste en la búsqueda de confirmar una sola hipótesis, prestando atención y concediendo relevancia a los factores que son compatibles con ella, sin repararse en los contrarios, que

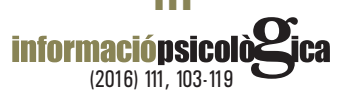


a su vez pudieran resultar compatibles con otras hipótesis alternativas. Cualquier proceso evaluativo que presente esta carencia no solo resulta incompleto,sino que además presenta un altísimo riesgo de llegar a conclusiones erróneas.

\section{2) Errores de interpretación}

\subsection{Errores asociados al evaluador.}

Prejuicios personales, sociales, culturales y cognitivos.

Los prejuicios, convencionalismos y creencias, incluso de forma no intencional, pueden influir en la evaluación e interpretación, llevando a la arbitrariedad de las conclusiones.Así, experiencias personales, creencias religiosas, estatus socio-económico y cultural, aprendizaje, prejuicios cognitivos, etc., pueden condicionar la objetividad de la evaluación en profesionales con poca experiencia y/o con falta de conocimiento exhaustivo del complejo proceso de la violencia de género. En este sentido, insistimos en realizar la evaluación mediante un protocolo que guíe la actuación evaluativa así como la conveniencia de dos evaluadores para aumentar la convergencia y la validez.

\section{Errores de atribución}

Encontramos que, por ejemplo, ante una situación de violencia más sutil, de tipo psicológico, se tiende a atribuir el maltrato a causas inestables, minimizando la importancia o gravedad del hecho, sin considerar sintomatología reactiva de las víctimas de malos tratos, de menor intensidad física. Otro error encontrado es el que podemos denominar error fundamental de atribución o sesgo de correspondencia (Gilbert, 1998) que consiste en la tendencia a explicar la conducta o comportamiento de una persona como adscrita a factores internos o rasgos de su personalidad, sin tener en cuenta la influencia de la situación, lo que puede llevar a interpretaciones descontextualizadas.

\section{Error de Otelo}

El error de Otelo da origen a errores de incredulidad en los que se incurre cuando se pasa por alto que una persona que está diciendo la verdad puede presentar el aspecto de una persona que miente si está sometida a tensión. Se olvida que una persona sincera puede estar asustada porque sospeche que no va a ser creída, por sus vivencias o por la propia situación evaluativa. El evaluador puede confundir esta situación con engaño o simulación, no dando credibilidad al testimonio.

Por otro lado, diversos estudios y meta-análisis revelan que no hay apenas indicadores conductuales o conductas no verbales que posibiliten diferenciar entre verdad y mentira. La evidencia muestra que los indicadores conductuales son escasos, poco diagnósticos y cambiantes (Masip y Herrero, 2015).

\section{Errores asociados a sesgos y mitos relacionados con la violencia machista.}

Los mitos relacionados con la violencia contra las mujeres, ampliamente descritos en la literatura sobre violencia con perspectiva de género, comportan una combinación de desinformación, creencias y actitudes que aún están vigentes socio-culturalmente y son aceptadas por muchos profesionales con falta de formación especializada y escaso juicio crítico.Desde el punto de vista jurídico,Díez (2015) lo describe de la siguiente manera:

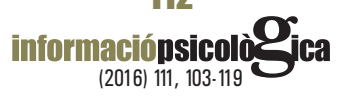


Algunos estereotipos (...) son: la víctima tiene que dar un perfil concreto, la edad de la víctima añade o resta credibilidad a la acusación, recuperarse del trauma de las agresiones (sean físicas, verbales, económicas, sexuales, estructurales o psíquicas, siendo el objetivo de todas ellas el sometimiento al control por parte del agresor) o haber iniciado una nueva relación de pareja también disminuye la veracidad de la declaración de la víctima-testigo (p.66).

Algunos sesgos pueden conducir en la situación evaluativa al victim blaming, inculpando a la víctima, más o menos sutilmente, de su propia victimización. Este tipo de distorsiones o sesgos no son puntuales ni unidimensionales, sino que forman parte de la propia disposición social (Loinaz, 2014) y también, por tanto, de algunos evaluadores, tal y como se desprende de algunas entrevistas e informes que hemos visto en nuestra práctica forense.

Asesoramiento, recomendaciones e intervención terapéutica por parte del/la forense

La función principal del psicólogo forense, con independencia del órgano judicial que solicite su intervención, será la evaluación y emisión de dictamen, actuando como perito. Su función es evaluativa, no terapéutica. Así debe aclararlo y no ofrecer conclusiones precipitadas, diagnósticos o recomendaciones clínicas a los sujetos objeto de evaluación forense, tal y como hemos constatado en algunas ocasiones.

\subsection{Errores asociados a la idiosincrasia de la persona evaluada}

Estilo de personalidad y estilo de comunicación

El patrón de comportamiento, estilo cognitivo, motivaciones y relaciones interpersonales de la persona evaluada puede comprometer la evaluación de la misma, pudiendo llevar a los evaluadores a interpretaciones erróneas. Por ejemplo, una persona con un estilo esquizoide de personalidad, puede mostrarse retraída, parca en palabras, no dará muchos detalles de los hechos, la comunicación puede ser poco fluida y escasa, y no mostrará afectividad o expresión emocional en relación a los hechos relatados. Todo ello puede influir negativamente, tanto en la evaluación diagnóstica como en la valoración sobre la credibilidad de su testimonio. Asimismo, el estilo de comunicación de una persona puede influir en los mensajes que recibe su evaluador, pudiendo interpretar su conducta como poco convincente o incluso como simulación o mentira, poniendo en duda toda la evaluación realizada.

Errores de atribución de la víctima

En base a la propia tolerancia cultural de la víctima, ésta puede minimizar, negar y omitir, de manera involuntaria, determinada información relativa a los malos tratos, por considerarla habitual y normalizada en su entorno socio-cultural, dificultando en este sentido la evaluación pericial. Por otra parte, la dependencia emocional de la mujer con respecto al denunciado está ampliamente explicada en la literatura psicológica y se relaciona con situaciones que pueden resultar contradictorias como la reanudación de la convivencia, retirada de la denuncia, negarse a referir información en contra de su pareja, nuevas interposiciones de denuncias, etc. (Ramos, Soler, Mayoy Garbizu, 2012). Este tipo de situaciones pueden confundir a los evaluadores condicionando sus conclusiones. 
Hay que tener en cuenta también las distorsiones cognitivas características de la mujer sometida a malos tratos continuados, tanto de tipo físico, psicológico sexual, encubiertos o manifiestos, tales como la minimización, negación o disociación, así como la aparente confusión e indiferencia, también asociada a un proceso de indefensión aprendida y compatibles con un trastorno por estrés postraumático en mujer maltratada. Así, por ejemplo, algunas mujeres evaluadas no informarán espontáneamente de determinadas conductas abusivas (por ejemplo, agresiones sexuales).

\section{3) Errores diagnósticos}

\subsection{Existen patologías que se dan en el contexto de maltrato que pueden derivar en equivocaciones diagnósticas.}

El sufrimiento psíquico consecuente de los malos tratos no es un problema de personalidad de la víctima, sino de las características inherentes al proceso de la violencia de género que produce una patología particular. Existe el riesgo, aún hoy todavía, y debido a los mitos culturales de la violencia contra la mujer, de que dichas mujeres víctimas sean diagnosticadas de trastornos de la personalidad, y descritas como dependientes, masoquistas o paranoides, culpabilizando y responsabilizándolas de sus propias secuelas (victim blaming). Este tipo de error puede estar también asociado a escaso conocimientode victimología forense en general, y de procesos de violencia de género en particular.

Para valorar el maltrato psicológico en este contexto se sugiere que su intensidad y severidad sea determinado en función tanto de su frecuencia como del impacto subjetivo que supone para la víctima (Ballester, y Villanueva, 2014): influencia de las variables edad, duración del maltrato y años de convivencia en el estado psicológico de los participantes, sobre todo en las mujeres.

A continuación, exponemos algunos ejemplos sobre este apartado que hemos encontrado en nuestra práctica profesional:

Es habitual en las mujeres víctimas de violencia de género desarrollar síntomas de trastorno por estrés postraumático (TEPT). Dentro de la sintomatología habitual de este cuadro clínico, se encuentra un estado de hipervigilancia que obliga a estar en alerta constante y prolongada, haciéndolas incluso reaccionar de forma exagerada a estímulos neutros, sintiéndose en un ambiente hostil y con una sensibilidad especial ante conductas y actitudes que han aprendido a interpretar como peligrosas para su integridad. Esta situación, erróneamente, se ha diagnosticado como "síndrome paranoide" o trastorno delirante en otros casos.

Las conductas de evitación del TEPT pueden ser confundidas con un trastorno de personalidad fóbico-evitativo, previo al maltrato, y por tanto no considerarse daño psíquico como consecuencia de sufrimiento de malos tratos.

3.2 Asumir que una víctima de maltrato tenga obligatoriamente que padecer secuelas en el momento de la evaluación.

La existencia de secuelas estará relacionada con diferentes variables, tales como la duración, frecuencia e intensidad de la violencia sufrida, el tiempo transcurrido en ausencia del elemento estresor, la capacidad de afrontamiento y recursos de la víctima, variables de 
personalidad, estrategias de reinterpretación positiva, apoyo profesional, el periodo transcurrido entre la ocurrencia del maltrato y el momento de la evaluación forense (a veces han pasado meses e incluso años), y otras variables. Hemos encontrado informes valorativos en los que la ausencia de secuelas en el momento de la evaluación ha llevado a concluir de forma causal la inexistencia de maltrato. La huella psíquica es un indicador del sufrimiento de malos tratos, no obstante, su ausencia no implica que dichos malos tratos no hayan tenido lugar.

\subsection{No valorar ni constatar posibles repercusiones psico-sociales en la víctima}

Se omite en algunos casos, en la valoración de secuelas, las repercusiones negativas a nivel familiar, social, laboral, no teniéndolas en cuenta y centrándose únicamente en aquellas secuelas clínicas significativas.

3.4 La ausencia de patología en el maltratador no implica ausencia de comportamiento violento.

Muchos agresores de género no presentan patología clínica. Los trastornos mentales en sentido estricto son poco frecuentes, pudiendo darse indicadores subclínicos. No debemos confundir las tipologías de maltratadores de género con un perfil previo que determine la comisión, o no, de un delito de maltrato. Existen factores de riesgo de violencia pero que son valorables una vez determinado el comportamiento violento.Este tipo de violencia tiene una causalidad compleja y multidimensional.

En informes sobre evaluación pericial en asuntos de violencia de género, hemos observado conclusiones en las que, no habiendo encontrado patrones violentos de personalidad o patología clínicamente significativa en los evaluados, se descarta la posibilidad de que estos sujetos puedan realizar conductas de maltrato.

3.5 No considerar prioritario la valoración de la víctima.

Para evaluar la violencia de género, desde el punto de vista victimológico, debemos centrarnos en el padecimiento y las consecuencias que para la víctima tiene y no tanto en la intencionalidad del agresor. Este sería otro proceso y otro momento evaluativo. La evaluación de malos tratos en el contexto de la violencia de género pasa obligatoriamente por la evaluación de la denunciante.

Se realizan habitualmente informes de acusados de malos tratos con el fin de valorar su estabilidad psicológica y equilibrio emocional como medio de prueba de su inocencia. Consideramos este tipo de prácticas un grave error pericial. Es por ello que se estima inconveniente establecer en los dictámenes periciales el nexo entre el equilibrio emocional y psicológico y la ausencia de comportamientos violentos.

Una valoración de predicción de riesgo de violencia, cuando se den las circunstancias en la situación pericial y el proceso judicial, será de utilidad para asesorar al juzgador en decisiones relacionadas con medidas de alejamiento, prisión preventiva o suspensión condicional de la pena, teniendo en cuenta el riesgo estimado de reincidencia futura del agresor (Andrés Pueyo y Echeburúa, 2010) 


\section{4) Errores de solicitud}

Un informe pericial se define y estructura, entre otras cosas, por el destinatario del mismo y la finalidad para la que se emite, respondiendo a unos extremos previamente solicitados. En este sentido hemos encontrado solicitudes mal planteadas e inviables que no posibilitan una adecuada respuesta desde el punto de vista de la propia ciencia psicológica.

Centrándonos en los asuntos de violencia de género, se nos ha solicitado por parte de las instancias judiciales, cuestiones tales como que se informe acerca de "valoración de la víctima y susceptibilidad de ser maltratada", "existencia de patología congruente con una persona violenta", "psicopatología del acusado a fin de determinar la ocurrencia de los hechos", etc.

Frente al dictamen de peritos, llegado el momento, los defensores de las partes, así como también los juzgadores, precisan entender e ilustrarse, de ahí la importancia de concretar de forma efectiva los extremos que dicha evaluación forense debe responder.

\section{DISCUSIÓN Y CONCLUSIONES}

Probablemente exista en la práctica pericial sobre violencia de género otros muchos errores que aquí no se han contemplado. Entendemos que, dada la complejidad de estos casos, los profesionales que realicen evaluaciones psicológicas en asuntos relacionados con la violencia de género deben de contar con unas características y aptitudes mínimas en cuanto a formación, especialización y experiencia. Es imprescindible, por tanto, no solo formación en Psicología Jurídica y Forense, sino también conocimientos amplios de psicopatología y victimología, formación específica en violencia de género, así como conocer y aplicar correctamente protocolos de evaluación específicos a fin de valorar secuelas en víctimas de violencia (Arce, Fariña, Carballal y Novo, 2009).

Se considera así mismo imprescindible, a fin de mejorar la calidad y eficacia de los informes periciales, unos conocimientos mínimos de la normativa legal (Sánchez, 2014; Cárdenas, 2011), entender las "reglas de juego" del contexto donde realizamos nuestro trabajo para poder ofrecer en nuestros peritajes información psicológica relevante y significativa en el contexto legal. En este sentido, como ejemplo y al hilo de lo anterior, en España ha entrado en vigor recientemente, en 28 de octubre de 2015 la nueva Ley del Estatuto de la Víctima (Ley 4/2015) donde se establecen normas mínimas sobre los derechos, apoyo y protección de las víctimas de delitos, incidiendo enel aspecto moral y reconociendo su dignidad (Vidal, 2015). Entendemos que estos aspectos también deben considerarse desde la Psicología Jurídica y Forense sin tener porqué suponer una dejación de funciones.

Otra conclusión que podemos extraer de nuestro análisis es el escaso, todavía, conocimiento y comprensión, por parte de diferentes profesionales en el contexto judicial, de las consecuencias de la violencia psicológica en el ámbito de la violencia de género. Contemplamos la importancia de la intervención psicológica jurídico-forense, que incluye el área pericial en materia tanto penal como civil, y de especial relevancia en el caso de víctimas vulnerables a quienes estas situaciones afectan de manera sustancial, por lo que habrá que adecuar una respuesta ad hoc que salvaguarde sus intereses. Así, en el caso de los menores como posibles víctimas de violencia de género es preciso considerar las variables que intervienen

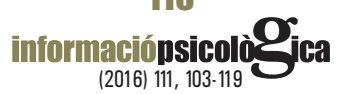


en la determinación del impacto emocional y/o la psicopatología consecuente (Olaya, Tarragona, de la Osa Chaparro y Ezpeleta, 2008) yentender cómo contribuyen a la adaptación psicológica de los niños diferentes aspectos, tales como el tipo de violencia, severidad, intensidad, frecuencia, continuidad, edad de inicio, relación con el agresor, relación con otras víctimas en el mismo contexto, o la concurrencia de diversos tipos de violencia (Kinard, 2004), y todo ello con el objeto de realizar una ajustada evaluación longitudinal y topográfica.

Conocer y entender la violencia psicológicay sus consecuencias en las víctimas nos llevaráa intervenir eficazmente como psicólogos forenses en el contexto judicial y a minimizar los errores de evaluación referidos a lo largo de nuestra exposición.

Abogamos por la utilización de protocolos evaluativos empíricamente avalados, eficaces, válidos y fiables que tengan en cuenta las diferentes áreas de evaluación, utilizando metodología rigurosa que minimice en lo posible los errores evaluativos. No menos importante es realizar informes periciales estructurados (Asensi, 2008, 2015) que trasladen de forma clara pero fundamentada la información que el perito psicólogo considere significativa y relevante para el caso que le ha sido encomendado.Uno de los protocolos recomendablesde evaluación adaptado a la práctica forense es el Sistema de Evaluación Global (Arce y Fariña, 2010, 2015) que consiste en un sistema categorial que integra unas fases ajustadas a casos de violencia de género y en el que se contempla tanto el testimonio como la huella psíquica, y cuyas implicaciones para la presentación del informe pretenden ajustarse en lo posible a los requerimientos de nuestro sistema de justicia.

Es así mismo imprescindible para el ejercicio profesional en este ámbito, reciclaje y formación continua para intervenir en estos asuntos de alta complejidad. El grado de fiabilidad que puede merecer un dictamen pericial vendrá ligado a los elementos y datos que el perito hubiera seleccionado para emitir su opinión técnica, así como de su comprensión del proceso que se está valorando.

Con todo lo referido y las propuestas de mejora, someramente mencionadas por economía espacial, se pretende llegar a cumplir realmente la función pericial de asesorar, científica y profesionalmente, a los tribunales de justicia, suministrando información objetiva, válida y fundamentada. Nuestro principal interés es que esta comunicación sirva como punto de reflexión acerca de la complejidad evaluativa que engloban estos asuntos y concienciar parallegar a una mayor profesionalización de todos los agentes implicados.

\section{REFERENCIAS}

Alcántara, M. V., López-Soler, C., Castro, M., \& López, J. J. (2013). Alteraciones psicológicas en menores expuestos a violencia de género: prevalencia y diferencias de género y edad. Anales de psicología, 29(3), 741-747.

Andrés Pueyo, A. (2012). Presente y futuro de la violencia interpersonal en las postrimerías del estado del bienestar. Anuario de psicología/The UB Journal of psychology, 42(2), 199-211.

Andrés-Pueyo, A. y Echeburúa, E. (2010). Valoración del riesgo de violencia: instrumentos disponibles e indicaciones de aplicación. Psicothema, 22(3), 403-409.

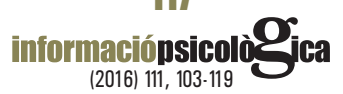


Arce, R. (2010). El Sistema de Evaluación Global en casos de violencia de género: Huella psíquica y testimonio. Informaciò Psicológica, (99), 19-35.

Arce, R., y Fariña, F. (2012). La entrevista psicológica forense a niños, adultos y discapacitados. Patología forense. Madrid: Editorial Bosch.

Arce, R. y Fariña, F. (2015). Evaluación psicológico-forense de la credibilidad y daño psíquico mediante el Sistema de Evaluación Global. En P. Rivas y G.L. Barrios (Dirs.), Violencia de género: Perspectiva multidisciplinar y práctica forense (pp. 411-441). Navarra: Thomson Aranzadi.

Arce, R, Fariña, F. Carballal, A. y Novo, M., 2009. Creación y validación de un protocolo de evaluación forense de las secuelas psicológicas de la violencia de género. Psicothema, 21(2), 241-247.

Asensi, L. F. (2008). La prueba pericial psicológica en asuntos de violencia de género. Revista Internauta de Pràctica Jurídica, (21), 15-29.

Asensi, L. F. y Díez, M. (2008). Errores más frecuentes en los peritajes psicológicos sobre malos tratos. $9^{\circ}$ Congreso Virtual de Psiquiatría. En Red [http://cdn.psiquiatria.com/bibliopsiquis/handle/10401/3952], Recuperado el 27 de mayo de 2016.

Asensi, L.F. (2014, Noviembre). Evaluación psicológica forense de menores en el ámbito de la violencia de género. Psicología forense, violencia de género y menores. IV Symposium Nacional Psicología Clínica y de la Salud con Niños y Adolescentes. Symposium organizado por Aitana Investigación. Universidad Miguel Hernández. Elche

Asensi, L.F. y Díez, M. (2015, Noviembre) Evaluación psicológica forense de menores en el ámbito de la violencia de género.Poster presentado en el 13 Congreso sobre la Violencia contra la Mujer de la Diputación de Alicante.

Ballester, A., y Villanueva, L. (2014). Valoración psicológica en delitos de violencia de género mediante el Inventario Clínico y Multiaxial de Millon III (MCMI-III). Anuario de Psicología Jurídica, 24(1), 9-18.

Cárdenas, A. E. M. (2011). La Victimología como estudio: redescubrimiento de la víctima para el proceso penal. $R e$ vista Prolegómenos. Derechos y Valores de la Facultad de Derecho, 14(27), 27-42.

Col.legi Oficial de Psicologia de Catalunya (2014). Guía de buenas prácticas para la evaluación psicológica forense y la práctica pericial.

Díez, P. I. (2015). La declaración de la perjudicada en los procedimientos de Violencia de Género: una aproximación crítica desde el ejercicio de la abogacía. Journal of Feminist, Gender and Women Studies, (01).

Muñiz, J., Hernández, A., y Ponsoda, V. (2015). Nuevas directrices sobre el uso de los tests: investigación, control de calidad y seguridad. Papeles del psicólogo, 36(3), 161-173.

Gilbert, D. T. (1998) Speeding with Ned: A personal view of the correspondence bias. In J. M. Darley \& J. Cooper (Eds.), Attribution and social interaction: The legacy of E. E. Jones. Washington, DC: APA Press.

Jouvencel, M. R. (2003) Por un protagonismo más activo del Juez en la valoración de la prueba pericial. Recuperado de: http://www.peritajemedicoforense.com/JOUVENCEL17.htm

Kinard, E.M. (2004). Methodological issues in assessing the effects of maltreatment characteristics on behavioral adjustment in maltreated children. Journal of Family Violence, 19, 303-318.

Labrador, F. J. ,Fernández-Velasco, M. R., \& Rincón, P. (2010). Características psicopatológicas de mujeres víctimas de violencia de pareja. Psicothema, 22 (1), 99-105.

Labrador, F.J., Paz Rincón, P., de Luis, P., Fernández-Velasco, R. (2004) Mujeres Víctimas de la violencia doméstica. Programas de Actuación. Madrid: Editorial Pirámide.

Larizgoitia, I. (2006). La violencia también es un problema de salud pública.Gaceta Sanitaria, 20, 63-70.

Ley del Estatuto de la víctima del delito (Ley4/2015, de 27 de abril). Boletín Oficial del Estado, no 101, 2015, de 28 de abril.

Ley Orgánica de Medidas de Protección Integral contra la Violencia de Género (LO 1/2004 de 28 de diciembre). Boletín Oficial del Estado, n³13, 2004, 29 de diciembre.

Loinaz, I. (2014). Distorsiones Cognitivas en Agresores de Pareja: Análisis de una herramienta de Evaluación. Terapia 
psicológica, 32(1), 5-17.

Lorente, M., Sánchez de Lara Sorzano, C., Naredo, C. (2006) Suicidio y Violencia de género. Federación de Mujeres Progresistas y Observatorio de Salud de la Mujer. Ministerio de Sanidad y Consumo.

Masip, J. y Herrero, C. (2015). Nuevas aproximaciones en detección de mentiras I.: antecedentes y marco teórico. Papeles del psicólogo, 36(2), 83-95.

Ministerio de Sanidad, Servicios Sociales e Igualdad de España. 2015. Macroencuesta de Violencia contra la Mujer 2015. Madrid: Delegación del Gobierno para la violencia de género. Secretaría de Estado de Servicios Sociales e Igualdad

Navarro Góngora, J., Navarro Abad, E., Vaquero, E., Carrascosa, A.M. (2004) Manual de Peritaje sobre malos tratos psicológicos. Valladolid: Junta de Castilla y León.

Novo, M., Seijo, D. 2009. "Aproximación psicosocial a la violencia de género: aspectos introductorios”. Pp. 36-74 en Violencia de género. Tratado psicológico y legal, editado por Fariña, F., Arce, R., Buela-Casal, G. Madrid: Biblioteca nueva.

Olaya, B., Tarragona, M. J., de la Osa Chaparro, N., \& Ezpeleta, L. (2008). Protocolo de evaluación de niños y adolescentes víctimas de la violencia doméstica. Papeles del psicólogo, 29(1), 123-135.)

OMS (2014) Informe sobre la situación mundial de la prevención de la violencia.

OMS (2016) Violencia contra la mujer. Nota descriptiva N. ${ }^{\circ}$ 239. [Documento WWW]. Url: http://www.who.int/mediacentre/factsheets/fs239/es/

ONU (2014), Guidelines for producing statistics on violence against women : Statistical surveys, ONU.

Porrúa, C., Rodríguez-Carballeria, A., Almendros, C., Escartín, J., Martín-Peña, J., \& Santaña, O. (2010). Análisis de las estrategias de abuso psicológico en la violencia de pareja. Informació Psicológica, (99), 53-63.

Pozueco, J. M. Moreno, J. M., Blázquez, M. y García-Baamonde, M.E. (2013). Psicópatas integrados/subclínicos en las relaciones de pareja: perfil, maltrato psicológico y factores de riesgo. Papeles del psicólogo, 34(1), 32-48.

Ramos, F. Á., Soler, C. B., Mayo, I. G., \& Garbizu, M. I. (2012). Variables relevantes en la evaluación forense integral de la violencia de género. Boletín criminológico (133), 1-4.

Sánchez, J. L. G. M. (2014). La Prueba Pericial en la Ley de Enjuiciamiento Civil. Balance Crítico. Revista Estudios Jurídicos. Segunda Época, (13).

Scott, M. T., y Manzanero, A.L. (2015). Análisis del expediente judicial: Evaluación de la validez de la prueba testifical. Papeles del psicólogo, 36(2), 139-144.

Vidal, A. (2015). El Estatuto de la víctima y la violencia de género. Recuperado de http://www.sepin.es/violenciadomestica/

Walker, L. E. (1989). Terrifying love: Why battered women kill and how society responds. New York: Harper \& Row Publishers

Walker, L. E. (2012). El síndrome de la mujer maltratada. Bilbao: Desclée de Brouwer. 